\title{
Rolf Griebel - Engagement in Gremien
}

\section{Gremien auf bayerischer Ebene}

\author{
Bayerisches Staatsministerium für Bildung und Kultus, Wissenschaft und Kunst/ \\ Bayerisches Staatsministerium für Wissenschaft, Forschung und Kunst \\ Zusammenarbeit der Länder Baden-Württemberg, Bayern und Sachsen im Bibliotheksbereich/ \\ Lenkungsgruppe \\ Zusammenarbeit der Länder Bayern und Nordrhein-Westfalen im Bibliotheksbereich/ \\ Lenkungsgruppe \\ Sachverständigenausschuss des Freistaats Bayern gemäß $§ 2$ Abs. 2 des Gesetzes zum Schutz \\ deutschen Kulturgutes gegen Abwanderung \\ Beirat des Hauses der Bayerischen Geschichte \\ Landesgeschichtliches Forum
}

Bayerisches Staatsministerium für Bildung und Kultus, Wissenschaft und Kunst/ Bayerisches Staatsministerium der Finanzen, für Landesentwicklung und Heimat bavarikon Rat

bavarikon Lenkungsausschuss

Qualitätszirkel: Zukunft der Fachhochschule für öffentliche Verwaltung und Rechtspflege in Bayern

\section{Bayerische Staatsbibliothek/Fachbehörde}

Konferenz der Direktoren der Universitätsbibliotheken (Vorsitz)

Konferenz der Leiter der Bibliotheken der Hochschulen für angewandte Wissenschaften (Vorsitz)

Konferenz der regionalen Staatlichen Bibliotheken (Vorsitz)

Kommission für EDV-Planung/IT-Beirat für das Bibliothekswesen in Bayern (Vorsitz)

Kommission für Erwerbung (Vorsitz)

Arbeitsgruppe für Erwerbungsautomatisierung

Arbeitsgruppe für Erwerbungskoordinierung

Arbeitsgruppe Personalbedarf

Arbeitsgruppe Bayerisches Etatmodell

Arbeitsgruppe Reform der Ausbildung von Diplombibliothekaren

\section{Bayerische Akademie der Wissenschaften}

Kommission für bayerische Landesgeschichte

\section{Münchener Zentrum für Editionswissenschaft Vorstand}

\section{Bayerische Beamtenfachhochschule}

Fachbereichskonferenz/Archiv- und Bibliothekswesen

\section{Bayerischer Bibliotheksverband}

Vorstand 


\author{
Bayerische Landesbibliothek Online (BLO) \\ Wissenschaftlicher Beirat
}

\author{
Verein der Förderer und Freunde der Bayerischen Staatsbibliothek \\ Vorstand
}

Freunde der Monacensia

Kuratorium

\title{
Gremien auf nationaler Ebene
}

\author{
Deutsche Forschungsgemeinschaft \\ Bibliotheksausschuss/Ausschuss für wissenschaftliche Bibliotheken und Informationssysteme \\ (zeitweise Vorsitz) \\ Unterausschuss für Sondersammelgebiete \\ Unterausschuss für die Förderung von Sondersammelgebiets- und Spezialbibliotheken \\ Unterausschuss für Überregionale Literaturversorgung \\ Arbeitsgruppe Koordinierung der Sondersammelgebiets- und Spezialbibliotheken \\ Arbeitsgruppe Projektkoordinierung der Virtuellen Fachbibliotheken \\ Projektbeirat WEBIS \\ Arbeitsgruppe EZUL-Projekt \\ Arbeitsgruppe Förderprogramm CD-ROM-Datenbanken \\ Arbeitsgruppe Elektronische Zeitschriften \\ Arbeitsgruppe Neustrukturierung der überregionalen Literaturversorgung
}

Wissenschaftsrat

Arbeitsgruppe Evaluierung der „Stiftung Weimarer Klassik und Kunstsammlungen“

\section{Stiftung Preußischer Kulturbesitz}

Beirat der Stiftung Preußischer Kulturbesitz (Vorsitz)

Bibliothekskommission des Beirats der Stiftung Preußischer Kulturbesitz (Vorsitz)

\section{Bundesministerium für Bildung und Wissenschaft}

Deutsch-deutsche Expertengruppe Bibliotheken/Bund-Länder-Arbeitsgruppe Bibliothekswesen

\section{Bundesministerium für Forschung und Technologie}

Projektbeirat DBV/OSI: Elektronischer Datenaustausch Buchhandel - Bibliotheken

\section{Hochschulrektorenkonferenz}

Arbeitsgruppe Beschaffung wissenschaftlicher Informationen - Neuausrichtung des Informationsund Publikationssystems der deutschen Hochschulen 
Arbeitsgemeinschaft Sammlung Deutscher Drucke

Direktorenkonferenz

Kompetenznetzwerk für Bibliotheken

Steuerungsgremium

Allianz zur Erhaltung des schriftlichen Kulturgutes

zeitweise Vorsitz

Deutsche Nationalbibliothek

Standardisierungsausschuss

Die Deutsche Bibliothek/Deutsche Nationalbibliothek

Beirat

\section{Deutsches Bibliotheksinstitut (DBI/EDBI)}

Kommission für die Erwerbung und Bestandsentwicklung

Expertengruppe Bestandsentwicklung in wissenschaftlichen Bibliotheken (Vorsitz)

Expertengruppe Erwerbungsprofile in universitären Bibliothekssystemen (Vorsitz)

Expertengruppe Etatverteilungsmodelle in universitären Bibliothekssystemen

Expertengruppe Inhaltliche Überarbeitung DBS, Teil B: Wissenschaftliche Bibliotheken

Expertengruppe Sicherung von Periodikabeständen

DigiZeitschriften e. V.

Vorstand

Bundesvereinigung Deutscher Bibliotheksverbände (BDB)/Börsenverein des

Deutschen Buchhandels

Arbeitsgruppe Empfehlungen für den Geschäftsverkehr zwischen Wissenschaftlichen Bibliotheken und dem Buchhandel

Arbeitsgruppe CD-ROM-Datenbanken

Arbeitsgruppe Elektronische Informationsnetzwerke und Datenbanken

Deutsches Institut für Normung (DIN)/Normenausschuss Bibliotheks- und

Dokumentationswesen (NABD)

Arbeitsausschuss 7, Bibliotheksmanagement

Sehepunkte - Rezensionsjournal für die Geschichtswissenschaften

Wissenschaftlicher Beirat

Gottfried-Wilhelm-Leibniz-Gemeinschaft

Evaluierungskommission Zentralbibliothek für Medizin (Köln) 
Internationale Jugendbibliothek

Stiftungsrat

Berlin-Brandenburgische Akademie der Wissenschaften

Betreuungskommission für das Akademienvorhaben „Jahresberichte für deutsche Geschichte“

Arbeitsgemeinschaft Historischer Forschungseinrichtungen (AHF) in der Bundesrepublik Deutschland e. V.

Ausschuss

Deutsches Museum München

Kuratorium

Gemeinsame Wissenschaftskonferenz (GWK)/Wissenschaftsgemeinschaft Gottfried Wilhelm Leibniz (WGL)

Steuerungsgremium der Kommission „Zukunft der Informationsinfrastruktur“

Arbeitsgruppe Retrodigitalisierung/Kulturelles Erbe (Vorsitz)

International Federation of Library Associations and Institutions (IFLA) in

Deutschland

Nationalkomitee

Deutsche Digitale Bibliothek

Vorstand

Max Weber Stiftung - Deutsche Geisteswissenschaftliche Institute im Ausland

Beirat für die Publikationsplattform perspectivia.net

Der Beauftragte der Bundesregierung für Kultur und Medien

Fachbeirat der Koordinierungsstelle zum Erhalt des schriftlichen Kulturguts (zeitweise Vorsitz)

Goethe-Institut

Beirat „Information und Bibliothek“

\section{Gremien auf internationaler Ebene}

International Federation of Library Associations and Institutions (IFLA)

Section on Acquisition and Exchange/Standing Committee

Section on Acquisition and Collection Development/Standing Committee 
Ligue des Bibliothèques Européennes de Recherche (LIBER)

Conspectus Group

Division for Library Management and Administration

Collection Development Executive Committee

Arbeitsgemeinschaft Konsortien/German, Austrian and Swiss Consortia Organization (GASCO)

Stellvertretender Vorsitzender 